\title{
Helical flows of fractional viscoelastic fluid in a circular pipe
}

\author{
Muzaffar Hussain Laghari, Kashif Ali Abro*, Asif Ali Shaikh
}

Department of Basic Sciences and Related Studies, Mehran University of Engineering Technology, Jamshoro, Pakistan

\section{A RTICLE INFO}

\section{Article history:}

Received 23 June 2017

Received in revised form

20 August 2017

Accepted 25 August 2017

Keywords:

Helical cylinder

Viscoelastic fluid

Fox H-function

Rheological impacts

\begin{abstract}
A B S T R A C T
The exploration of this study is devoted to investigate the helical effects for the flow of fractionalized viscoelastic fluid in helically moved cylinder. The cylinder starts to oscillate and rotate about its axis when $t=0^{+}$with velocities. By applying mathematical transforms (Hankel and discrete Laplace transforms) exact solutions are found out for velocities and shear stresses. The general solutions satisfy initial conditions $u_{1}(r, 0)=u_{2}(r, 0)=$ $\frac{\partial u_{1}(r, 0)}{\partial t}=\frac{\partial u_{2}(r, 0)}{\partial t}=0, \quad$ as well as boundary conditions $u_{1}(R, t)=$ $R \Omega H(t) \sin (\omega t)$ or $\cos (\omega t), \quad$ and $\quad u_{2}(R, t)=U H(t) \sin \omega t / \cos \omega t$. The solutions are presented in terms of series form and expressed in terms of generalized Fox $H$-function $H_{j, k+1}^{1, j}(Z)$. Special cases have been traced out for non-Newtonian fluids (fractional and ordinary Second Grade, fractional and ordinary Newtonian fluid and ordinary Maxwell Fluid). Three types of fluid models are presented for rheological comparison, namely (i) fractional and ordinary Maxwell fluid, (ii) fractional and ordinary second grade fluid and (iii) fractional and ordinary Newtonian fluid. Finally, the rheology is influenced with distinct parameters and material limitations for helically moved cylinder by depicting graphical analysis.
\end{abstract}

(C) 2017 The Authors. Published by IASE. This is an open access article under the CC BY-NC-ND license (http://creativecommons.org/licenses/by-nc-nd/4.0/).

\section{Introduction}

The practical applications of flow of nonNewtonian fluids lie among the modern industries. Such industries have diverted the attention of engineers, mathematicians and scientists for the solutions of flow problems of non-Newtonian fluids. Various rheological materials like polymer melts, suspensions, clay coatings, drilling mud, elastomers, certain greases and oils, and numerous emulsions are considered as non-Newtonian fluids. In order to exhibit certain characteristics of non-Newtonian fluids there is not at least single constitutive equation, this is due to complex behavior of fluid. The rheology of non-Newtonian fluids has distinct characteristics in fermentation, boiling, polymer processing, molten plastic foam processing, composite processing many others (Fetecau and Corina, 2005; Fetecau et al., 2007; Erdogan and Imrak, 2005; Chen et al., 2004; Hayat et al., 2004a; 2004b; Abro and Shaikh, 2015). Nowadays, viscoelastic fluid (Maxwell model) is acknowledged by many scientists and engineers in several engineering and industrial processes. This is due to

\footnotetext{
* Corresponding Author.

Email Address: kashif.abro@faculty.muet.edu.pk (K. A. Abro) https://doi.org/10.21833/ijaas.2017.010.014

2313-626X/C 2017 The Authors. Published by IASE.

This is an open access article under the CC BY-NC-ND license

(http://creativecommons.org/licenses/by-nc-nd/4.0/)
}

involvement of viscoelastic material, for instance, glues, paints, melts of polymers, asphalts, biological solutions, colloids and several others. In continuation, Hayat et al. (2006) investigated flow problem for second grade fluid in cylindrical geometry in which they traced out analytical solutions. Analytical solutions have been obtained by Akl (2014) on the structure of stretching cylinder for unsteady boundary flow. Altintas and Ozkol (2015) analyzed non-heated and heated cases in circular pipes for magnetohydrodynamic flow. Sulochana and Sandeep (2016) worked at different temperature for shrinking cylinder with heat transfer behavior of magnetohydrodynamics. Masood et al. (2016) investigated a stagnation-point flow with the nonlinear radiative on Sisko fluid over stretching cylinder. They established numerical solutions via shooting method through forth order Runge-Kutta method by transforming governing partial differential equation of stretching cylinder. They also explored nonlinear Rosseland approximation and effects of thermal radiation. Jamil et al. (2011) analyzed longitudinal and torsional constantly an infinite accelerated cylinder with for second order liquid. They found exact analytical solutions for shear stress and velocity profile. Mahmood et al. (2010) worked on the annular region of cylinders for generalized second order liquid with oscillatory flow. They utilized integral transform to investigate some exact analytical solutions with few limiting 
cases. Siddique and Vieru (2009) examined circular cylinder for rotational fluid of second order liquid and investigated analytical study in cylindrical configuration. Abdulhameed et al. (2016) perceived oscillating flow in circular cylinder for heat performance and compared due to different pressure waveforms. Sulochana and Sandeep (2016) studied heat transfer and momentum behavior of few nanoparticles embedded towards porous cylinder. They obtained numerical solutions by employing Runge-Kutta Felhberg technique. Of course the list of study on circular cylinder for viscoelastic fluid can be continuous but we end it with some recent references (Shah and Qi, 2010; Wang and $\mathrm{Xu}, 2009$; Nazar et al., 2010; Fetecau et al., 2010; Malekzadeh et al., 2011; Abro and Solangi, 2017; Muhammad et al., 2015; Abro, 2016; Rostami et al., 2014; Rashidi et al., 2014; Rashidi et al., 2012; Rashidi et al., 2015). Motivating by above studies, our purpose is to investigate the helical effects for the flow of fractionalized viscoelastic fluid in helically moved cylinder. The cylinder starts to oscillate and rotate about its axis when $t=0^{+}$with velocities. By applying mathematical transforms (Hankel and discrete Laplace transforms) exact solutions are found out for velocities and shear stresses. The solutions are presented in terms of series form and expressed in terms of generalized Fox $\mathrm{H}$-function $H_{j, k+1}^{1, j}(Z)$. Special cases have been traced out for non-Newtonian fluids (fractional and ordinary Second Grade, fractional and ordinary Newtonian fluid and ordinary Maxwell Fluid). Three types of fluid models are presented for rheological comparison, namely (i) fractional and ordinary Maxwell fluid, (ii) fractional and ordinary second grade fluid and (iii) fractional and ordinary Newtonian fluid.. Finally, the rheology is influenced with distinct parameters and material limitations among two helically moved cylinders by depicting graphical analysis.

\section{Mathematical modeling of helices}

The Cauchy stress tensor $T$ is an incompressible Maxwell fluid is given (Fetecau et al., 2010; Abro, 2016)

$\mathrm{T}=-p \mathrm{I}+\mathrm{S}, \mathrm{S}+\Lambda\left(\dot{\mathrm{S}}-\mathrm{LS}-\mathrm{SL}^{\mathrm{T}}\right)=\mu \mathrm{A}$,

where $-p \mathrm{I}, \mathrm{S}, \quad \mathrm{L}, \quad \mathrm{A}=\mathrm{L}+\mathrm{L}^{\mathrm{T}}, \mu, \quad \Lambda, \quad T$ are the indeterminate spherical stress due to the constraint of incompressibility, the extra-stress tensor, velocity gradient, first Rivilvin Ericksen tensor, dynamic viscosity, relaxation time, transpose operation. This Maxwell model can also be characterized for Newtonian fluid by letting $\Lambda \rightarrow 0$. The microscopic polymers and their predictions of the normal-stress differences are also characterized by this model. Due to this significance, this model is useful to analyze dilute polymeric fluids in viscoelasticity. Here velocity field is assumed as

$u=u(r, t)=u_{1}(r, t) e_{\theta}+u_{2}(r, t) e_{z}, \quad \mathrm{~S}=\mathrm{S}(r, t)$, where, $\boldsymbol{e}_{\theta}$ and $\boldsymbol{e}_{z}$ are unit vectors in the $\theta$ and $z$ direction. For such flows the constraint of incompressibility is automatically satisfied. If the fluid is at rest up to the moment $t=0$ then

$u(r, 0)=\mathrm{S}(r, 0)=0$,

and implementing $S_{r r}=0$ in Eq. 1 then we arrived at meaning full equations as defined below

$\Lambda \frac{\partial}{\partial t} \tau_{1}(r, t)-\mu \frac{\partial u_{2}(r, t)}{\partial r}+\mu \frac{u_{2}(r, t)}{r}+\tau_{1}(r, t)=0$,

$\Lambda \frac{\partial}{\partial t} \tau_{2}(r, t)-\mu \frac{\partial u_{2}(r, t)}{\partial t}+\tau_{2}(r, t)=0$,

here, $\tau_{1}=S_{r \theta}$ and $\tau_{2}=S_{r z}$ are the shear stresses. While, due to nonappearance of pressure gradient, balance of linear momentum and ignoring body the forces lead the following equation for symmetry of rotation as

$\rho \frac{\partial u_{1}(r, t)}{\partial t}-\frac{\partial \tau_{1}(r, t)}{\partial r}-\frac{2 \tau_{1}(r, t)}{r}=0$,

$\rho \frac{\partial u_{2}(r, t)}{\partial t}-\frac{\partial \tau_{2}(r, t)}{\partial r}-\frac{\tau_{2}(r, t)}{r}=0$,

eliminating $\tau_{1}$ and $\tau_{2}$ from Eqs. 4-7, we arrive at the equations governs the helical flow Fetecau et al. (2008)

$\frac{\partial u_{1}(r, t)}{\partial t}+\Lambda \frac{\partial}{\partial t}\left(\frac{\partial u_{1}(r, t)}{\partial t}\right)-v\left(\frac{\partial^{2}}{\partial r^{2}}+\frac{1}{r} \frac{\partial}{\partial r}-\frac{1}{r^{2}}\right) u_{1}(r, t)=$

$0, t>0$

$\frac{\partial u_{2}(r, t)}{\partial t}+\Lambda \frac{\partial}{\partial t}\left(\frac{\partial u_{2}(r, t)}{\partial t}\right)-v\left(\frac{\partial^{2}}{\partial r^{2}}+\frac{v}{r} \frac{\partial}{\partial r}\right) u_{2}(r, t)=0, \quad t>0$,

where $v=\frac{\mu}{\rho}$ is the kinematic viscosity of the fluid. Meanwhile, we consider here fractional Maxwell fluid at rest in an oscillating circular cylinder of radius $R$. At time $t=0^{+}$the cylinder begins to rotate about its own axis $(t=0)$ with the angular velocity $\Omega \sin (\omega t)$ or $\Omega \cos (\omega t)$ and oscillates along the same axis with $U \sin (\omega t)$ or $U \cos (\omega t)$. Due to shear the fluid is gradually moved and its velocity being of the pattern as in (2), while the governing equations are (4-5) and (8-9). Such flow produces helicity, this is due to fact that the streamline of helicity are helices as shown in Fig. 1. The conditions are:

- Initial condition:

$u_{1}(r, 0)=u_{2}(r, 0)=\tau_{1}(r, 0)=\tau_{2}(r, 0)=\frac{\partial u_{1}(r, 0)}{\partial t}=$
$\frac{\partial u_{2}(r, 0)}{\partial t}=0$,

- Boundary condition for angular velocity:

$u_{1}(R, t)=R \Omega H(t) \sin (\omega t)$ or $R \Omega H(t) \cos (\omega t), t \geq 0$

$u_{2}(R, t)=U H(t) \sin (\omega t)$ or $U H(t) \cos (\omega t), \quad t \geq 0^{\prime}$

where $H(t)$ is Heaviside function. In order to develop the governing equations for helical flow (45) and (8-9) in terms of non-integer order derivative, we implement the Caputo-fractional operator, we get $\frac{\partial u_{1}(r, t)}{\partial t}+\Lambda D_{t}^{\chi}\left(\frac{\partial u_{1}(r, t)}{\partial t}\right)-v\left(\frac{\partial^{2}}{\partial r^{2}}+\frac{1}{r} \frac{\partial}{\partial r}-\frac{1}{r^{2}}\right) u_{1}(r, t)=$

$0, t>0$, 
$\frac{\partial u_{2}(r, t)}{\partial t}+\Lambda D_{t}^{\chi}\left(\frac{\partial u_{2}(r, t)}{\partial t}\right)-v\left(\frac{\partial^{2}}{\partial r^{2}}+\frac{v}{r} \frac{\partial}{\partial r}\right) u_{2}(r, t)=0, \quad t>$ 0 ,

$\Lambda D_{t}^{\chi} \tau_{1}(r, t)-\mu \frac{\partial u_{1}(r, t)}{\partial r}+\mu \frac{u_{1}(r, t)}{r}+\tau_{1}(r, t)=0$,

$\Lambda D_{t}^{\chi} \tau_{2}(r, t)-\mu \frac{\partial u_{2}(r, t)}{\partial t}+\tau_{2}(r, t)=0$, where, $D_{t}^{\chi}$ represent the non-integer order Caputo fractional operator defined as (Abro et al., 2016; Abro et al., 2017a)

$D_{t}^{\chi} u(t)=\left\{\begin{array}{lc}\frac{1}{\Gamma(1-\chi)} \int_{0}^{t} \frac{u^{\prime}(q)}{(t-q)^{\chi}} d q, & 0<\chi<1 ; \\ \frac{d u(t)}{d t}, & \chi=1\end{array}\right.$,

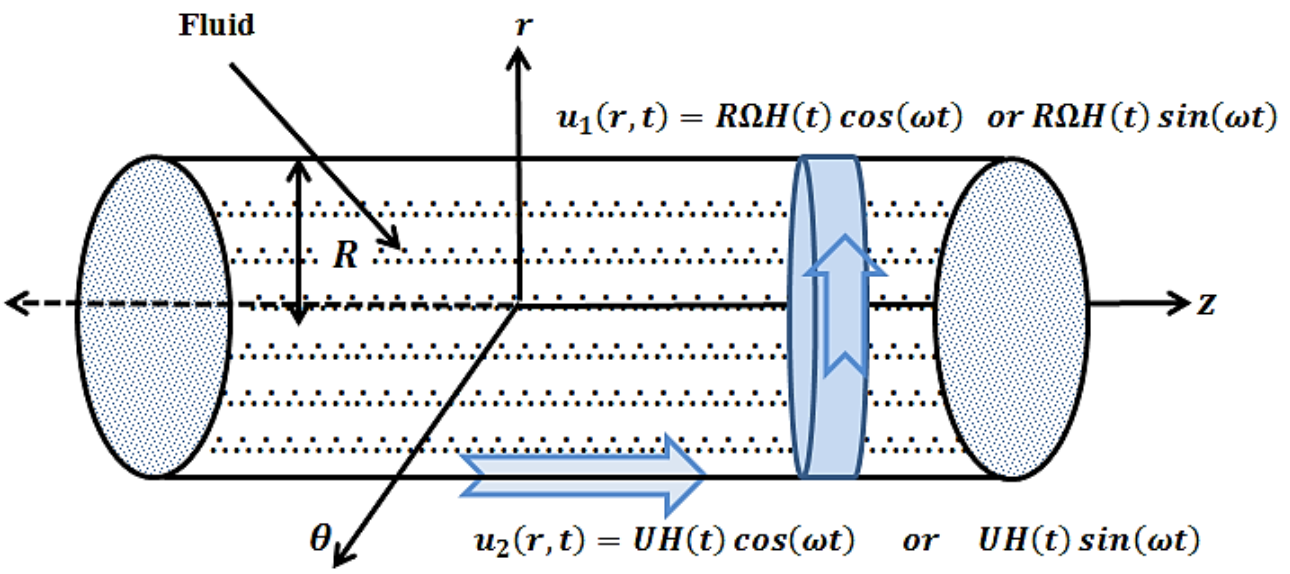

Fig. 1: Geometrical configuration of helecity

\section{Solution of the problem}

\subsection{Velocity field}

- Case-I: For sine oscillations: Applying Laplace transform on Eqs. 12-13 and keeping in mind Eqs. 10-11, we arrive at

$\left(s+\Lambda^{\chi} s^{1+\chi}\right) \bar{u}_{1}(r, s)=v\left(\frac{\partial^{2}}{\partial r^{2}}+\frac{1}{r} \frac{\partial}{\partial r}-\frac{1}{r^{2}}\right) \bar{u}_{1}(r, s)$,
$\left(s+\Lambda^{\chi} s^{1+\chi}\right) \bar{u}_{2}(r, s)=v\left(\frac{\partial^{2}}{\partial r^{2}}+\frac{1}{r} \frac{\partial}{\partial r}\right) \bar{u}_{2}(r, s)$,

where the conditions $\quad \bar{u}_{1}(r, s)=\frac{R \Omega \omega}{\left(s^{2}+\omega^{2}\right)} \quad$ and $\bar{u}_{2}(r, s)=\frac{U \omega}{\left(s^{2}+\omega^{2}\right)} \quad$ are to satisfy Eqs. 17-18. Employing finite Hankel transform on Eqs. 17-18 and using Appendix A (A1 and A3) on Eq. 17 and (A2, A4) on Eq. 18, we get

$$
\begin{aligned}
& \bar{u}_{1 H}\left(r_{\alpha}, s\right)=\frac{\Omega J_{2}\left(R r_{\alpha}\right) \omega v R^{2} r_{\alpha}}{s^{2}\left(\Lambda^{\chi_{S}}{ }^{1+\chi}+s+v r_{\alpha}^{2}\right)+\omega^{2}\left(\Lambda \chi_{S^{1}}{ }^{1+\chi}+s+v r_{\alpha}^{2}\right)^{\prime}} \\
& \bar{u}_{2 H}\left(r_{\beta}, s\right)=\frac{U J_{1}\left(R r_{\beta}\right) \omega v R r_{\beta}}{s^{2}\left(\Lambda \chi_{S^{1+\chi}}+s+v r_{\beta}^{2}\right)+\omega^{2}\left(\Lambda \chi_{S^{1+\chi}}+s+v r_{\beta}^{2}\right)^{\prime}}
\end{aligned}
$$

in order to satisfy imposed conditions, we present suitable equivalent forms of Eqs. 19-20 as

$$
\begin{aligned}
& \bar{u}_{1 H}\left(r_{\alpha}, s\right)=\frac{\Omega J_{2}\left(R r_{\alpha}\right) \omega R^{2}}{r_{\alpha}\left(s^{2}+\omega^{2}\right)}- \\
& \frac{\Omega J_{2}\left(R r_{\alpha}\right) R^{2}}{r_{\alpha}} \frac{\omega\left(\Lambda^{\chi} s^{1+\chi}+s\right)}{s^{2}\left(\Lambda^{\chi} s^{1+\chi}+s+v r_{\alpha}^{2}\right)+\omega^{2}\left(\Lambda_{S^{1+\chi}}+s+v r_{\alpha}^{2}\right)^{\prime}}
\end{aligned}
$$$$
\bar{u}_{2 H}\left(r_{\beta}, s\right)=\frac{U J_{1}\left(R r_{\beta}\right) \omega R}{r_{\beta}\left(s^{2}+\omega^{2}\right)}-
$$$$
\frac{U J_{1}\left(R r_{\beta}\right) R}{r_{\beta}} \frac{\omega\left(s+\Lambda^{\chi} S^{1+\chi}\right)}{s^{2}\left(\Lambda^{\chi_{S}}{ }^{1+\chi}+s+v r_{\beta}^{2}\right)+\omega^{2}\left(\Lambda \chi^{1+\chi}+s+v r_{\beta}^{2}\right)^{\prime}}
$$

applying inverse Hankel transform on Eqs. 21-22 and using Appendix A (A5) and (A6), we get suitable series expansion as

$$
\bar{u}_{1}(r, s)=\frac{r \Omega \omega}{s^{2}+\omega^{2}}-\frac{2 \Omega \omega}{s^{2}+\omega^{2}} \sum_{\alpha=1}^{\infty} \frac{J_{1}\left(r r_{\alpha}\right)}{r_{\alpha} J_{2}\left(R r_{\alpha}\right)}
$$

$$
\begin{aligned}
& \sum_{s_{1}=0}^{\infty}\left(-v r_{\alpha}^{2}\right)^{s_{1}} \sum_{S_{2}=0}^{\infty} \frac{(-\Lambda \chi)^{s_{2}} \Gamma\left(s_{1}+s_{2}\right)}{s_{2} ! \Gamma\left(s_{1}\right) s^{s_{1}-s_{2} \chi}}, \\
& \bar{u}_{2}(r, s)=\frac{U \omega}{s^{2}+\omega^{2}}-\frac{2 U \omega}{R\left(s^{2}+\omega^{2}\right)} \sum_{\beta=1}^{\infty} \frac{J_{0}\left(r r_{\beta}\right)}{r_{\beta} J_{1}\left(R r_{\beta}\right)} \\
& \sum_{s_{1}=0}^{\infty}\left(-v r_{\alpha}^{2}\right)^{s_{1}} \sum_{s_{2}=0}^{\infty} \frac{(-\Lambda \chi)^{s_{2}} \Gamma\left(s_{1}+s_{2}\right)}{s_{2} ! \Gamma\left(s_{1}\right) s^{s_{1}-s_{2} \chi}}
\end{aligned}
$$

inverting Eqs. 23-24 by means of Laplace transform and using theorem of convolution product, we have final form of velocities in terms of Fox- $\mathrm{H}$ function as

$u_{1}(r, t)=r \Omega H(t) \sin (\omega t)-$

$2 \Omega H(t) \sum_{\alpha=1}^{\infty} \frac{J_{1}\left(r r_{\alpha}\right)}{r_{\alpha} J_{2}\left(R r_{\alpha}\right)} \sum_{s_{1}=0}^{\infty}\left(-v r_{\alpha}^{2}\right)^{s_{1}} \int_{0}^{t} \sin \omega(t-\delta) \times$

$\mathrm{H}_{1,3}^{1,1}\left[\left.\left(-\frac{\Lambda^{\chi}}{t \chi}\right)^{s_{2}}\right|_{(0,1),\left(1-s_{1}, 0\right),\left(1-s_{1},-\chi\right)} ^{\left(1-s_{1}, 1\right)}\right] d \delta$,

$u_{2}(r, t)=U H(t) \sin (\omega t)-$

$\frac{2 U H(t)}{R} \sum_{\beta=1}^{\infty} \frac{J_{0}\left(r r_{\beta}\right)}{r_{\beta} J_{1}\left(R r_{\beta}\right)} \sum_{S_{1}=0}^{\infty}\left(-v r_{\beta}^{2}\right)^{s_{1}} \int_{0}^{t} \sin \omega(t-\delta) \times$

$H_{1,3}^{1,1}\left[\left.\left(-\frac{\Lambda^{\chi}}{t \chi}\right)^{s_{2}}\right|_{(0,1),\left(1-s_{1}, 0\right),\left(1-s_{1},-\chi\right)} ^{\left(1-s_{1}, 1\right)}\right] d \delta$

where, $H_{j, k+1}^{1, j}(Z)$ is generalized Fox $\mathrm{H}$-function defined as (Abro et al., 2017b; Abro et al., 2017c)

$t^{n} \sum_{p}^{\infty} \frac{(-\Phi)^{p} \prod_{k=1}^{m} \Gamma\left(i_{k}+I_{k} p\right)}{p ! \prod_{k=1}^{n} \Gamma\left(j_{k}+J_{k} p\right)}=$
$\boldsymbol{H}_{m, n+1}^{1, m}\left[\Phi \mid \begin{array}{c}\left(1-i_{1}, I_{1}\right),\left(1-i_{2}, I_{2}\right), \ldots,\left(1-i_{m}, I_{M}\right) \\ (0,1),\left(1-j_{1}, J_{1}\right),\left(1-j_{2}, J_{2}\right), \ldots,\left(1-j_{n}, J_{N}\right)\end{array}\right]$.

\subsection{Shear stress}

Applying Laplace transform on Eqs. 14-15 and keeping in mind Eqs. 10-11, we arrive at

$$
\begin{aligned}
& \bar{\tau}_{1}(r, s)=\mu\left(1+\Lambda^{\chi} s^{\chi}\right)^{-1}\left(\frac{\partial \bar{u}_{1}(r, s)}{\partial r}-\frac{\bar{u}_{1}(r, s)}{r}\right), \\
& \bar{\tau}_{2}(r, s)=\mu\left(1+\Lambda^{\chi} s^{\chi}\right)^{-1} \frac{\partial \bar{u}_{2}(r, s)}{\partial r},
\end{aligned}
$$

substituting Appendix A (A7) and (A8) and using facts of Bessel's function $J_{0}\left(r r_{\alpha}\right)=-r_{\alpha} J_{1}\left(r r_{\alpha}\right)$ also $r r_{\beta} J_{1}^{\prime}\left(r r_{\beta}\right)-J_{1}\left(r r_{\beta}\right)=-r r_{\beta} J_{2}\left(r r_{\beta}\right)$ in Eqs. 28-29, we obtain simplified form as 
$\bar{\tau}_{1}(r, s)=2 \mu \Omega \omega \sum_{\alpha=1}^{\infty} \frac{J_{2}\left(r r_{\alpha}\right)}{J_{2}\left(R r_{\alpha}\right)} \sum_{s_{1}=0}^{\infty}\left(-v r_{\alpha}^{2}\right)^{s_{1}}$

$\sum_{s_{3}=0}^{\infty}\left(-\Lambda^{\chi}\right)^{s_{3}} \sum_{s_{2}=0}^{\infty} \frac{\left(-\Lambda^{\chi}\right)^{s_{2}} \Gamma\left(s_{1}+s_{2}\right) \omega}{s_{2} ! \Gamma\left(s_{1}\right)\left(s^{2}+\omega^{2}\right) s^{s_{1}-s_{2} \chi-s_{3} \chi}}$

$\bar{\tau}_{2}(r, s)=\frac{2 \mu U \omega}{R} \sum_{\beta=1}^{\infty} \frac{J_{1}\left(r r_{\beta}\right)}{J_{1}\left(R r_{\beta}\right)} \sum_{s_{1}=0}^{\infty}\left(-v r_{\beta}^{2}\right)^{s_{1}}$

$\sum_{s_{3}=0}^{\infty}\left(-\Lambda^{\chi}\right)^{s_{3}} \sum_{s_{2}=0}^{\infty} \frac{\left(-\Lambda^{\chi}\right)^{s_{2}} \Gamma\left(s_{1}+s_{2}\right) \omega}{s_{2} ! \Gamma\left(s_{1}\right)\left(s^{2}+\omega^{2}\right) s^{s_{1}-s_{2} \chi-s_{3} \chi}}$,

inverting Eqs. 30-31 by means of Laplace transform and using theorem of convolution product, we have final form of shear stresses in terms of Fox-H function as

$\tau_{1}(r, t)=2 \Omega H(t) \omega \mu \sum_{\alpha=1}^{\infty} \frac{J_{2}\left(r r_{\alpha}\right)}{J_{2}\left(R r_{\alpha}\right)} \sum_{s_{1}=0}^{\infty}\left(-v r_{\alpha}^{2}\right)^{s_{1}}$

$\sum_{s_{3}=0}^{\infty}\left(-\Lambda^{\chi}\right)^{s_{3}} \int_{0}^{t} \sin \omega(t-\delta) \times$

$\mathbf{H}_{1,3}^{1,1}\left[\left.\left(-\frac{\Lambda^{\chi}}{t^{\chi}}\right)^{s_{2}}\right|_{(0,1),\left(1-s_{1}, 0\right),\left(1-s_{1},-\chi\right)} ^{\left(1-s_{1}, 1\right)}\right] d \delta$

$\tau_{2}(r, t)=\frac{2 U H(t) \omega \mu}{R} \sum_{\beta=1}^{\infty} \frac{J_{1}\left(r r_{\beta}\right)}{J_{1}\left(R r_{\beta}\right)} \sum_{S_{1}=0}^{\infty}\left(-v r_{\alpha}^{2}\right)^{s_{1}}$

$\sum_{s_{3}=0}^{\infty}\left(-\Lambda^{\chi}\right)^{s_{3}} \int_{0}^{t} \sin \omega(t-\delta) \times$

$\mathbf{H}_{1,3}^{1,1}\left[\left.\left(-\frac{\Lambda^{\chi}}{t \chi}\right)^{s_{2}}\right|_{(0,1),\left(1-s_{1}, 0\right),\left(1-s_{1},-\chi\right)} ^{\left(1-s_{1}, 1\right)}\right] d \delta$.

- Case-II: For cosine oscillations: Implementing identical algorithm, we also investigated the analytical solutions of cosine oscillations as

$u_{1}(r, t)=r \Omega H(t) \cos (\omega t)-$

$2 \Omega H(t) \sum_{\alpha=1}^{\infty} \frac{J_{1}\left(r r_{\alpha}\right)}{r_{\alpha} J_{2}\left(R r_{\alpha}\right)} \sum_{s_{1}=0}^{\infty}\left(-v r_{\alpha}^{2}\right)^{s_{1}} \int_{0}^{t} \cos \omega(t-\delta) \times$

$\mathbf{H}_{1,3}^{1,1}\left[\left.\left(-\frac{\Lambda^{\chi}}{t \chi}\right)^{s_{2}}\right|_{(0,1),\left(1-s_{1}, 0\right),\left(1-s_{1},-\chi\right)} ^{\left(1-s_{1}, 1\right)}\right] d \delta$.

$u_{2}(r, t)=U H(t) \cos (\omega t)-$

$\frac{2 U H(t)}{R} \sum_{\beta=1}^{\infty} \frac{J_{0}\left(r r_{\beta}\right)}{r_{\beta} J_{1}\left(R r_{\beta}\right)} \sum_{S_{1}=0}^{\infty}\left(-v r_{\beta}^{2}\right)^{s_{1}} \int_{0}^{t} \cos \omega(t-\delta) \times$

$\boldsymbol{H}_{1,3}^{1,1}\left[\left.\left(-\frac{\Lambda^{\chi}}{t \chi}\right)^{s_{2}}\right|_{(0,1),\left(1-s_{1}, 0\right),\left(1-s_{1},-\chi\right)} ^{\left(1-s_{1}, 1\right)}\right] d \delta$

$\tau_{1}(r, t)=$

$2 \Omega H(t) \omega \mu \sum_{\alpha=1}^{\infty} \frac{J_{2}\left(r r_{\alpha}\right)}{J_{2}\left(R r_{\alpha}\right)} \sum_{s_{1}=0}^{\infty}\left(-v r_{\alpha}^{2}\right)^{s_{1}} \sum_{s_{3}=0}^{\infty}\left(-\Lambda^{\chi}\right)^{s_{3}} \int_{0}^{t} \sin \omega(t-$ $\delta) \times \mathbf{H}_{1,3}^{1,1}\left[\left.\left(-\frac{\Lambda^{\chi}}{t \chi}\right)^{s_{2}}\right|_{(0,1),\left(1-s_{1}, 0\right),\left(1-s_{1},-\chi\right)} ^{\left(1-s_{1}, 1\right)}\right] d \delta$,

$\tau_{2}(r, t)=$

$\frac{2 U H(t) \omega \mu}{R} \sum_{\beta=1}^{\infty} \frac{J_{1}\left(r r_{\beta}\right)}{J_{1}\left(R r_{\beta}\right)} \sum_{S_{1}=0}^{\infty}\left(-v r_{\alpha}^{2}\right)^{s_{1}} \sum_{S_{3}=0}^{\infty}\left(-\Lambda^{\chi}\right)^{s_{3}} \int_{0}^{t} \sin \omega(t-$

$\delta) \times \mathbf{H}_{1,3}^{1,1}\left[\left.\left(-\frac{\Lambda^{\chi}}{t \chi}\right)^{s_{2}}\right|_{(0,1),\left(1-s_{1}, 0\right),\left(1-s_{1},-\chi\right)} ^{\left(1-s_{1}, 1\right)}\right] d \delta$.

\section{Special solutions}

\subsection{Solutions of Maxwell fluid for ordinary differential operator}

Case-I: For sine oscillations: In order to retrieve the solutions for sine and cosine oscillations, we substitute $\chi \rightarrow 1$ in Eqs. 25-26, 34-35 and Eqs. 32-33, 36-37, we arrive

$u_{1}(r, t)=r \Omega H(t) \sin (\omega t)-$

$2 \Omega H(t) \sum_{\alpha=1}^{\infty} \frac{J_{1}\left(r r_{\alpha}\right)}{r_{\alpha} J_{2}\left(R r_{\alpha}\right)} \sum_{S_{1}=0}^{\infty}\left(-v r_{\alpha}^{2}\right)^{s_{1}} \int_{0}^{t} \sin \omega(t-\delta) \times$

$\mathbf{H}_{1,3}^{1,1}\left[\left.\left(-\frac{\Lambda}{t}\right)^{s_{2}}\right|_{(0,1),\left(1-s_{1}, 0\right),\left(1-s_{1},-1\right)} ^{\left(1-s_{1}, 1\right)}\right] d \delta$, $u_{2}(r, t)=U H(t) \sin (\omega t)-$

$\frac{2 U H(t)}{R} \sum_{\beta=1}^{\infty} \frac{J_{0}\left(r r_{\beta}\right)}{r_{\beta} J_{1}\left(R r_{\beta}\right)} \sum_{S_{1}=0}^{\infty}\left(-v r_{\beta}^{2}\right)^{s_{1}} \int_{0}^{t} \sin \omega(t-\delta) \times$

$\boldsymbol{H}_{1,3}^{1,1}\left[\left.\left(-\frac{\Lambda}{t}\right)^{s_{2}}\right|_{(0,1),\left(1-s_{1}, 0\right),\left(1-s_{1},-1\right)} ^{\left(1-s_{1}, 1\right)}\right] d \delta$.

$\tau_{1}(r, t)=$

$2 \Omega H(t) \omega \mu \sum_{\alpha=1}^{\infty} \frac{J_{2}\left(r r_{\alpha}\right)}{J_{2}\left(R r_{\alpha}\right)} \sum_{s_{1}=0}^{\infty}\left(-v r_{\alpha}^{2}\right)^{s_{1}} \sum_{S_{3}=0}^{\infty}(-\Lambda)^{s_{3}} \int_{0}^{t} \sin \omega(t-$

$\delta) \times \mathbf{H}_{1,3}^{1,1}\left[\left.\left(-\frac{\Lambda}{t}\right)^{s_{2}}\right|_{(0,1),\left(1-s_{1}, 0\right),\left(1-s_{1},-1\right)} ^{\left(1-s_{1}, 1\right)}\right] d \delta$,

$\tau_{2}(r, t)=$

$\frac{2 U H(t) \omega \mu}{R} \sum_{\beta=1}^{\infty} \frac{J_{1}\left(r r_{\beta}\right)}{J_{1}\left(R r_{\beta}\right)} \sum_{S_{1}=0}^{\infty}\left(-v r_{\alpha}^{2}\right)^{s_{1}} \sum_{s_{3}=0}^{\infty}(-\Lambda)^{s_{3}} \int_{0}^{t} \sin \omega(t-$

$\delta) \times \mathbf{H}_{1,3}^{1,1}\left[\left.\left(-\frac{\Lambda}{t}\right)^{S_{2}}\right|_{(0,1),\left(1-s_{1}, 0\right),\left(1-s_{1},-1\right)} ^{\left(1-s_{1}, 1\right)}\right] d \delta$.

\section{- Case-II: For cosine oscillations}

$u_{1}(r, t)=r \Omega H(t) \cos (\omega t)-$

$2 \Omega H(t) \sum_{\alpha=1}^{\infty} \frac{J_{1}\left(r r_{\alpha}\right)}{r_{\alpha} J_{2}\left(R r_{\alpha}\right)} \sum_{s_{1}=0}^{\infty}\left(-v r_{\alpha}^{2}\right)^{s_{1}} \int_{0}^{t} \cos \omega(t-\delta) \times$

$\mathbf{H}_{1,3}^{1,1}\left[\left.\left(-\frac{\Lambda}{t}\right)^{s_{2}}\right|_{(0,1),\left(1-s_{1}, 0\right),\left(1-s_{1},-1\right)} ^{\left(1-s_{1}, 1\right)}\right] d \delta$,

$u_{2}(r, t)=U H(t) \cos (\omega t)-$

$\frac{2 U H(t)}{R} \sum_{\beta=1}^{\infty} \frac{J_{0}\left(r r_{\beta}\right)}{r_{\beta} J_{1}\left(R r_{\beta}\right)} \sum_{S_{1}=0}^{\infty}\left(-v r_{\beta}^{2}\right)^{s_{1}} \int_{0}^{t} \cos \omega(t-\delta) \times$

$\boldsymbol{H}_{1,3}^{1,1}\left[\left.\left(-\frac{\Lambda}{t}\right)^{s_{2}}\right|_{(0,1),\left(1-s_{1}, 0\right),\left(1-s_{1},-1\right)} ^{\left(1-s_{1}, 1\right)}\right] d \delta$.

$\tau_{1}(r, t)=$

$2 \Omega H(t) \omega \mu \sum_{\alpha=1}^{\infty} \frac{J_{2}\left(r r_{\alpha}\right)}{J_{2}\left(R r_{\alpha}\right)} \sum_{S_{1}=0}^{\infty}\left(-v r_{\alpha}^{2}\right)^{s_{1}} \sum_{S_{3}=0}^{\infty}(-\Lambda)^{s_{3}} \int_{0}^{t} \sin \omega(t-$

$\delta) \times \mathbf{H}_{1,3}^{1,1}\left[\left.\left(-\frac{\Lambda}{t}\right)^{s_{2}}\right|_{(0,1),\left(1-s_{1}, 0\right),\left(1-s_{1},-1\right)} ^{\left(1-s_{1}, 1\right)}\right] d \delta$

$\tau_{2}(r, t)$

$=\frac{2 U H(t) \omega \mu}{R} \sum_{\beta=1}^{\infty} \frac{J_{1}\left(r r_{\beta}\right)}{J_{1}\left(R r_{\beta}\right)} \sum_{s_{1}=0}^{\infty}\left(-v r_{\alpha}^{2}\right)^{s_{1}} \sum_{s_{3}=0}^{\infty}(-\Lambda)^{s_{3}} \int_{0}^{t} \sin \omega(t$

$-\delta)$

$\times \mathbf{H}_{1,3}^{1,1}\left[\left.\left(-\frac{\Lambda}{t}\right)^{s_{2}}\right|_{(0,1),\left(1-s_{1}, 0\right),\left(1-s_{1},-1\right)} ^{\left(1-s_{1}, 1\right)}\right] d \delta$.

\subsection{Solutions of Newtonian fluid}

- Case-I: For sine oscillations: In order to retrieve the solutions for sine and cosine oscillations, we substitute $\Lambda \rightarrow 0$ in Eqs. 25-26, 34-35 and Eqs. 3233, 36-37, we obtained Newtonian solutions

$u_{1}(r, t)=r \Omega H(t) \sin (\omega t)-$

$2 \Omega \omega H(t) \sum_{\alpha=1}^{\infty} \frac{J_{1}\left(r r_{\alpha}\right)}{r_{\alpha} J_{2}\left(R r_{\alpha}\right)} \int_{0}^{t} \sin \omega(t-\delta) \operatorname{Exp}\left(-v r_{\alpha}^{2}\right) t d \delta$,

$u_{2}(r, t)=U H(t) \sin (\omega t)-$

$\frac{2 U \omega H(t)}{R} \sum_{\beta=1}^{\infty} \frac{J_{0}\left(r r_{\beta}\right)}{r_{\beta} J_{1}\left(R r_{\beta}\right)} \int_{0}^{t} \sin \omega(t-\delta) \operatorname{Exp}\left(-v r_{\alpha}^{2}\right) t d \delta$,

$\tau_{1}(r, t)=2 \mu \Omega \omega H(t) \sum_{\alpha=1}^{\infty} \frac{J_{2}\left(r r_{\alpha}\right)}{J_{2}\left(R r_{\alpha}\right)} \int_{0}^{t} \sin \omega(t-$

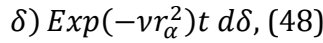

$\tau_{2}(r, s)=\frac{2 \mu U \omega H(t)}{R} \sum_{\beta=1}^{\infty} \frac{J_{1}\left(r r_{\beta}\right)}{J_{1}\left(R r_{\beta}\right)} \int_{0}^{t} \sin \omega(t-$

ס) $\operatorname{Exp}\left(-v r_{\alpha}^{2}\right) t d \delta$

(49)

\section{- Case-II: For cosine oscillations}

$u_{1}(r, t)=r \Omega H(t) \cos (\omega t)-$

$2 \Omega H(t) \sum_{\alpha=1}^{\infty} \frac{J_{1}\left(r r_{\alpha}\right)}{r_{\alpha} J_{2}\left(R r_{\alpha}\right)} \int_{0}^{t} \cos \omega(t-\delta) \operatorname{Exp}\left(-v r_{\alpha}^{2}\right) t d \delta$ 
$u_{2}(r, t)=U H(t) \cos (\omega t)-$

$\frac{2 U H(t)}{R} \sum_{\beta=1}^{\infty} \frac{J_{0}\left(r r_{\beta}\right)}{r_{\beta} J_{1}\left(R r_{\beta}\right)} \int_{0}^{t} \cos \omega(t-\delta) \operatorname{Exp}\left(-v r_{\alpha}^{2}\right) t d \delta$,

$\tau_{1}(r, t)=2 \mu \Omega H(t) \sum_{\alpha=1}^{\infty} \frac{J_{2}\left(r r_{\alpha}\right)}{J_{2}\left(R r_{\alpha}\right)} \int_{0}^{t} \cos \omega(t-$

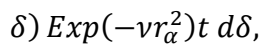

$\tau_{2}(r, s)=\frac{2 \mu U H(t)}{R} \sum_{\beta=1}^{\infty} \frac{J_{1}\left(r r_{\beta}\right)}{J_{1}\left(R r_{\beta}\right)} \int_{0}^{t} \cos \omega(t-$

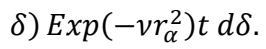

It is also worth pointed out that our general solutions can also be retrieved for ordinary Newtonian fluid when relaxation time $\left(\Lambda^{\chi}=0\right)$ is zero and fractional parameter $(\chi=1)$ is assumed to be equal to one Fetecau et al. (2008). In continuation, the solution obtained by Fetecau et al. (2008) can be retrieved by substituting $\omega=0$ in Eqs. 46-49 or Eqs. 50-53.

\section{Numerical results and discussions}

In this portion, our purpose is to analyze few rheological parameters for helical flow of fractionalized viscoelastic fluid in helically moved cylinder numerically. The cylinder starts to oscillate and rotate about its axis with angular as well as oscillating velocities corresponding with shear stresses.

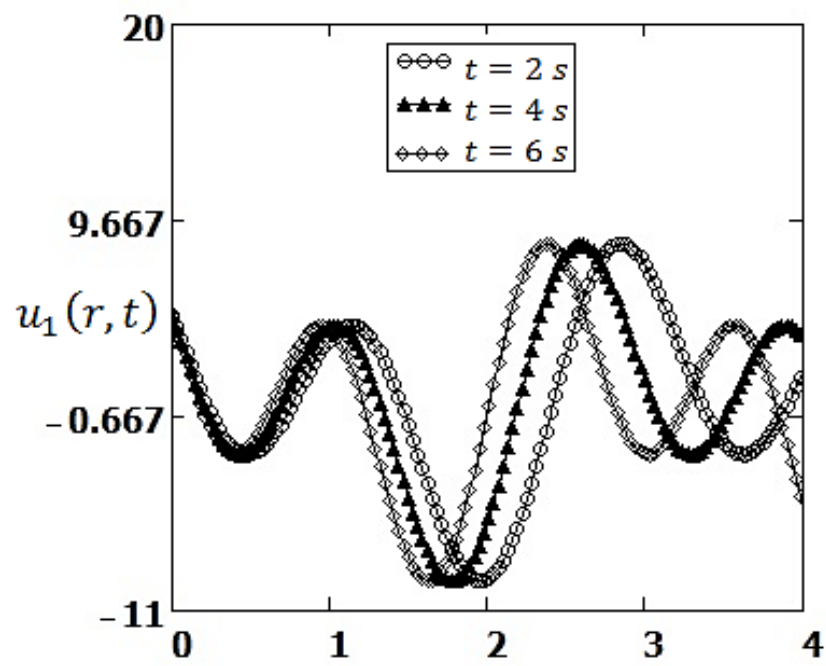

Fig. 2: Plot of angular and oscillating velocities for $t$

Fig. 3 is depicted to show the effects of viscosity parameter $v$ on angular velocity $u_{1}(r, t)$ and oscillating velocity $u_{2}(r, t)$. It is noticed that that as viscosity increase; both velocities have oscillating behavior of fluid flow in scattering manners. It is also pointed out that oscillating velocity $u_{2}(r, t)$ has dominant behavior of flow in comparison with angular velocity $u_{1}(r, t)$.

- Fig. 4 is plotted for amplitude $\omega$ of angular velocity $u_{1}(r, t)$ and oscillating velocity $u_{2}(r, t)$, as expected the periodic response of fluid flow over the
The exact solutions are investigated for both velocities and shear stresses along with imposed conditions $u_{1}(r, 0)=u_{2}(r, 0)=\frac{\partial u_{1}(r, 0)}{\partial t}=\frac{\partial u_{2}(r, 0)}{\partial t}=0$ and $u_{1}(R, t)=R \Omega H(t) \sin (\omega t)$ or $\cos (\omega t)$, and $u_{2}(R, t)=U H(t) \sin \omega t / \cos \omega t$. The general solutions are presented in terms Fox $\mathbf{H}$-function $\boldsymbol{H}_{j, k+1}^{1, j}(Z)$ with few particular cases, namely fractionalized second grade, ordinary second grade, ordinary Maxwell fluid and Newtonian fluid. Under these circumstances, the rheology is considered with distinct parameters and material limitations.

In order to have an insight of physical interpretation for helically moved cylinder, the graphical analysis is depicted for knowing the hidden differences and similarities on fluid flow. However, the major outcomes are enumerated below:

- Fig. 2 is prepared for the influences of time parameter on angular velocity $u_{1}(r, t)$ and oscillating velocity $u_{2}(r, t)$. Both velocities have qualitatively identical behavior for increase in time $t$. It is found that angular velocity $u_{1}(r, t)$ has sequestrating behavior and oscillating velocity $u_{2}(r, t)$ has smattering behavior on the whole domain of cylinder surface.

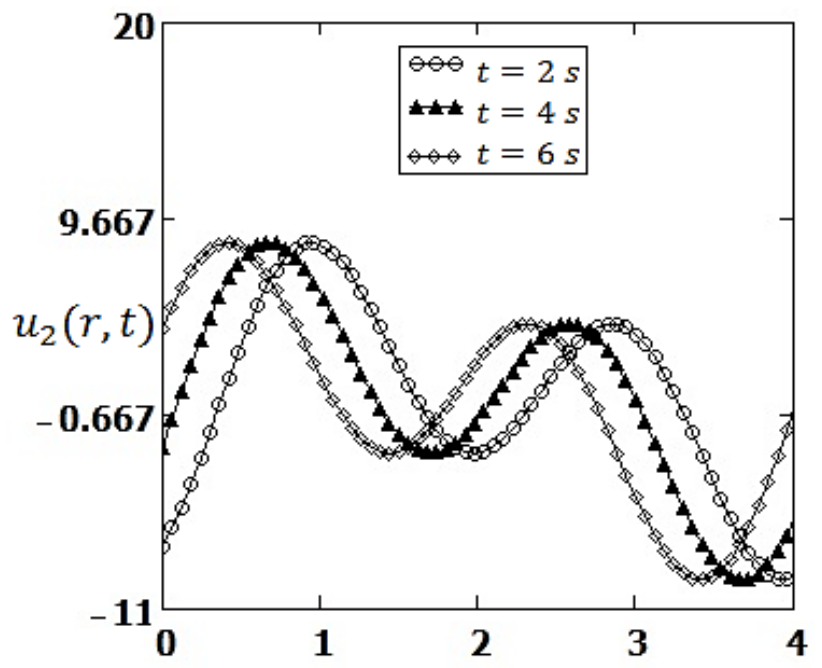

boundary of circular cylinder is observed. It is also clear that both velocities have distinct fluctuations; this may be due to the fact of imposed boundary conditions.

- The variation of radius of circular cylinder is displayed in Fig. 5 with range $0.1,0.2,0.3$. It is noted that both velocities have oscillating behavior within insignificant interval. Also, it is observed that an angular velocity has shorter oscillations in comparison with oscillating velocity. 

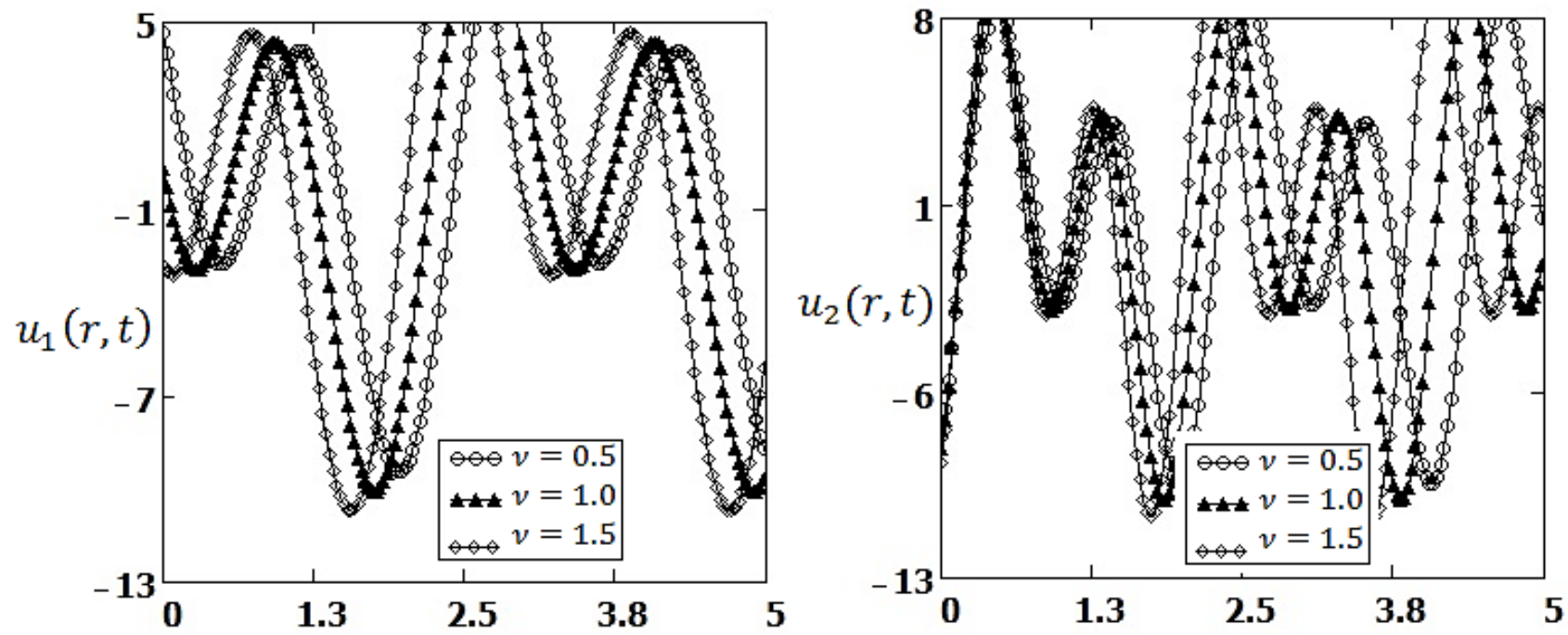

Fig. 3: Plot of angular and oscillating velocities for $v$
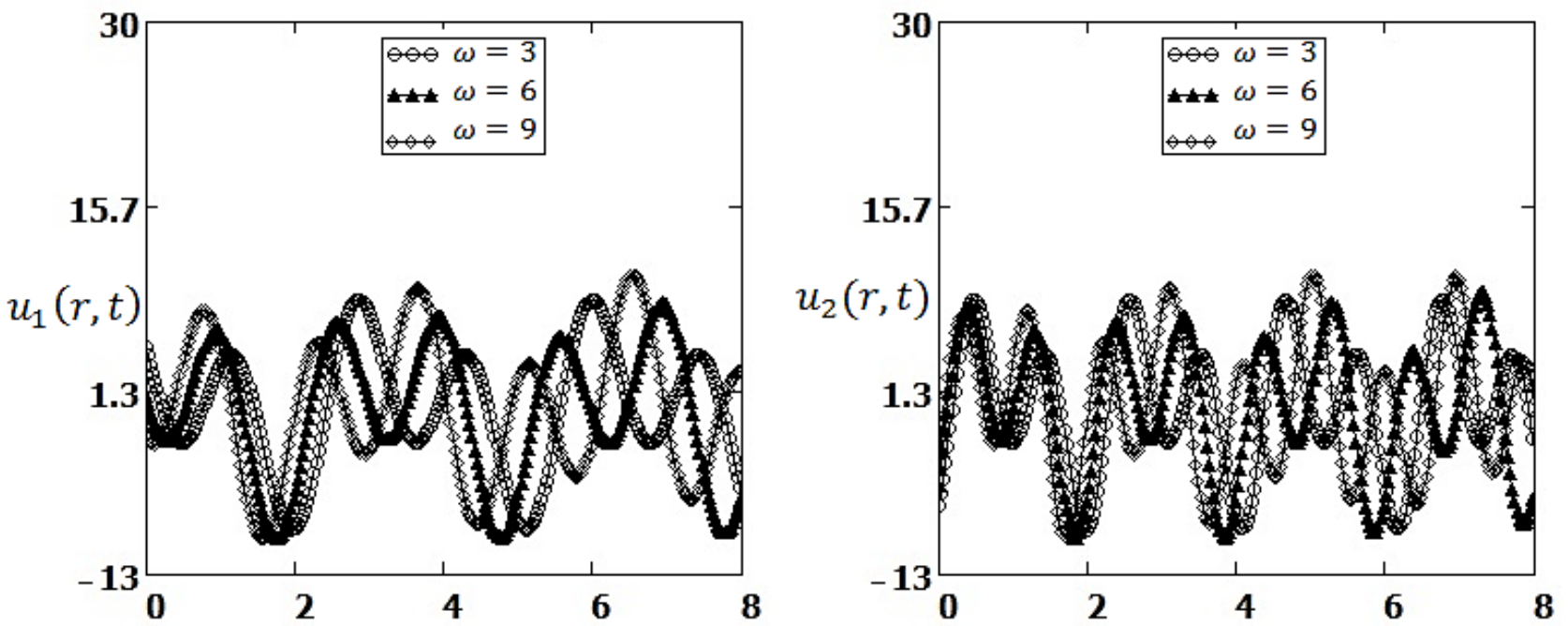

Fig. 4: Plot of angular and oscillating velocities for $\omega$
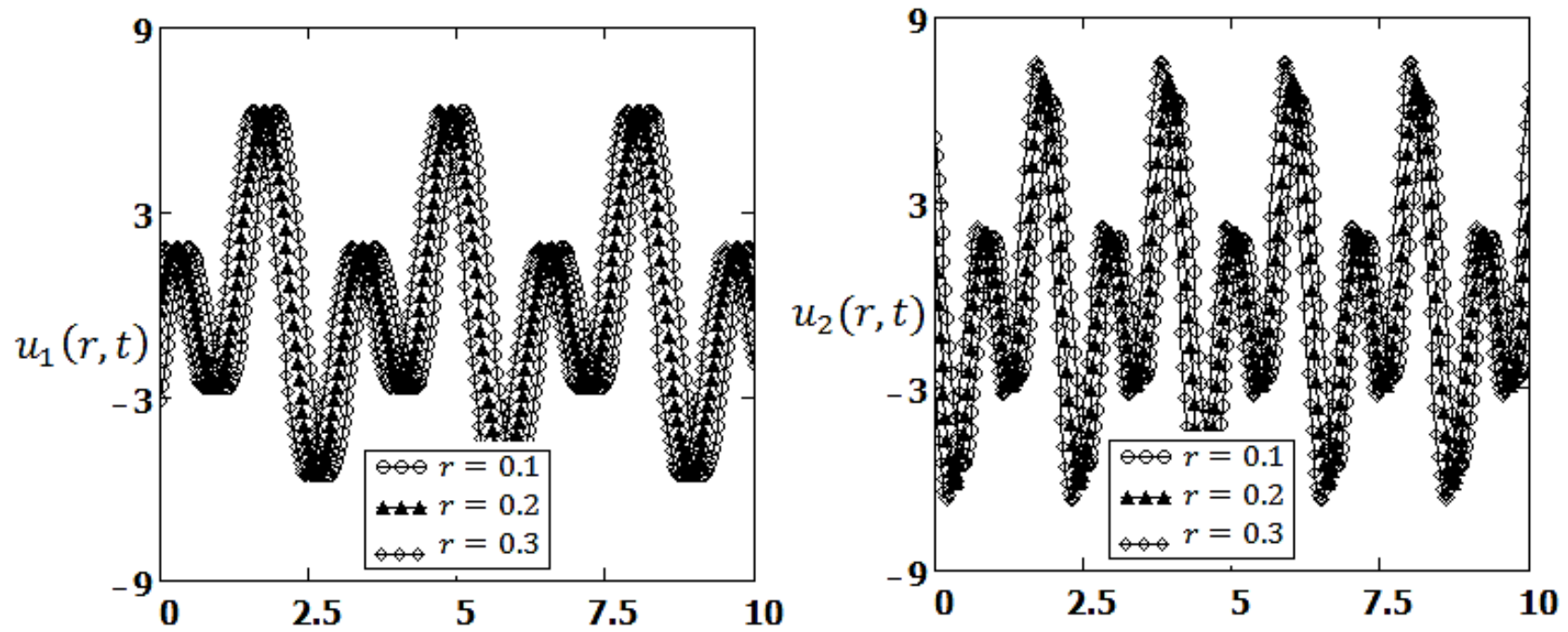

Fig. 5: Plot of angular and oscillating velocities for $r$

- Fig. 6 explains the hidden phenomenon of fractional parameter $\chi$ on both angular and oscillating velocities. Here, the behavior of both velocities is quite identical to each other. It is pointed out that oscillating velocity $u_{2}(r, t)$ moves rapidly in comparison with angular velocity $u_{1}(r, t)$. This may be due to fact of fractional order derivatives which examines the complete description of the memory effectively.

- Figs. 7 and 8 are drawn for the comparison of three ordinary as well as fractional models i-e (i) fractional and ordinary Maxwell fluid, (ii) fractional and ordinary second grade fluid and (iii) fractional and ordinary Newtonian fluid. In both figures, 
angular velocity $u_{1}(r, t)$ and oscillating velocity $u_{2}(r, t)$ has opposite trend for fluid flow. It is observed that ordinary Newtonian fluid moves faster in angular velocity $u_{1}(r, t)$ and ordinary Maxwell moves faster in oscillating velocity. On the other hand, fractional Newtonian fluid moves faster in angular velocity $u_{1}(r, t)$ and fractional

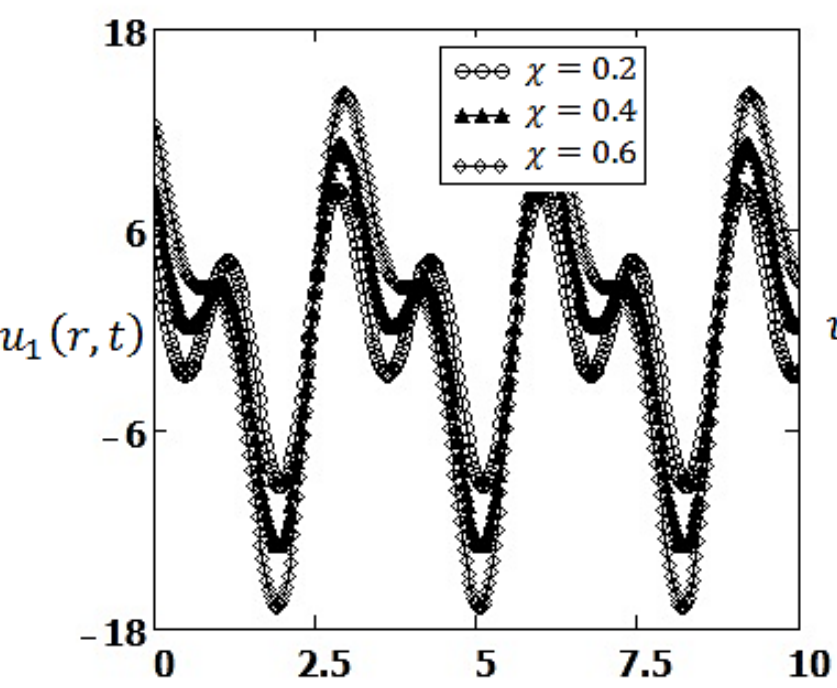

Fig. 6: Plot of angular and oscillating velocities for $\chi$
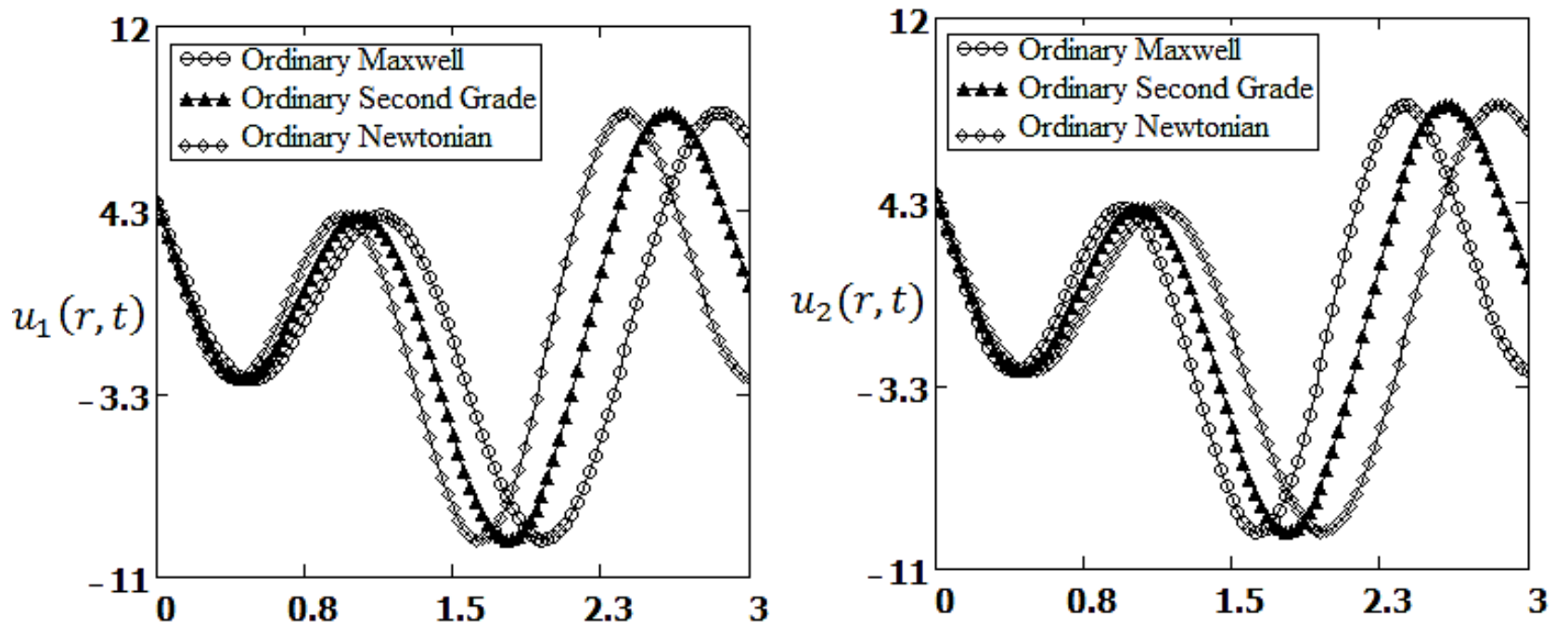

Fig. 7: Comparison of angular and oscillating velocities for three ordinary models
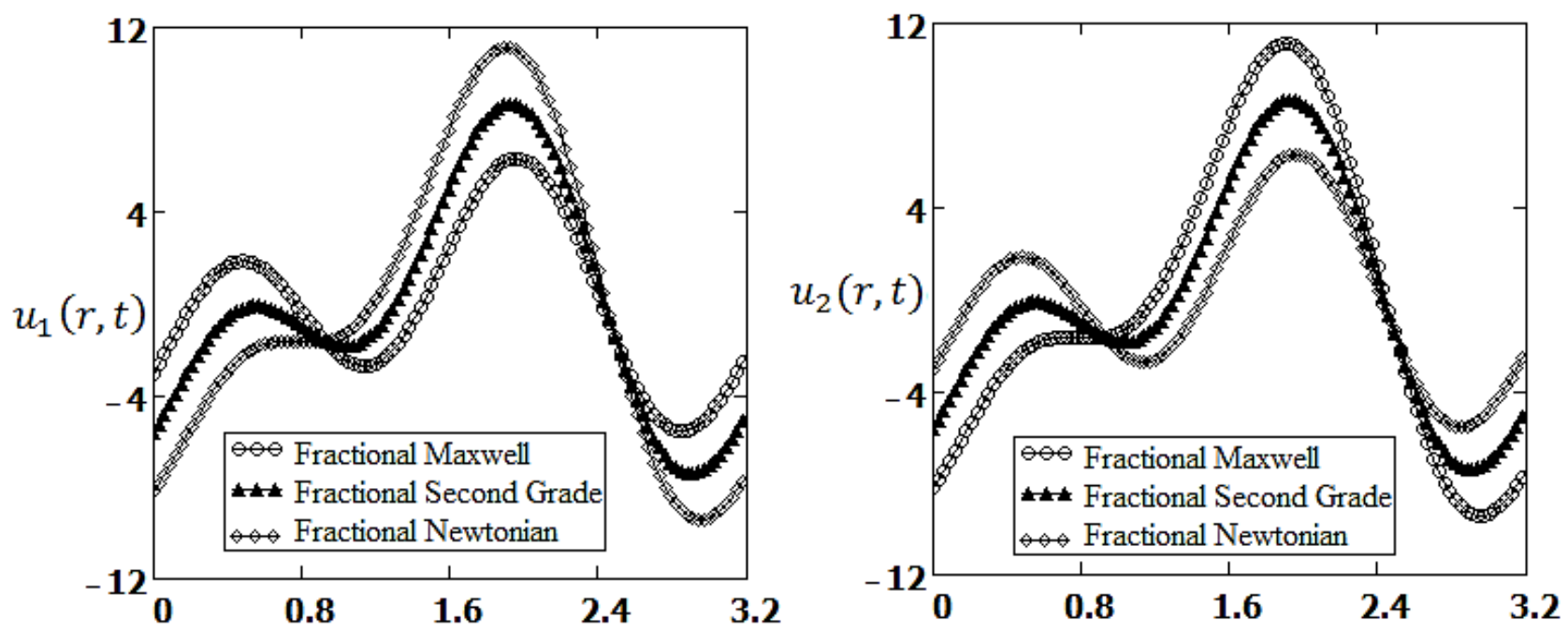

Fig. 8: Comparison of angular and oscillating velocities for three fractional models 


\section{Acknowledgement}

The authors are highly thankful and grateful to Mehran university of Engineering and Technology,

\section{List of symbols}

$\begin{array}{ll}u_{1}(r, t) & \text { Angular velocity }\left(m \cdot s^{-1}\right) \\ \tau_{1}(r, t) \text { or } \tau_{2}(r, t) \text { Shear stress } \\ D_{t}^{\chi} & \text { Caputo fractional derivative } \\ T & \text { Cauchy stress tensor } \\ A & \text { Rivilvin Ericksen tensor } \\ r, \theta, z & \text { Cylindrical coordinates }(m) \\ -p I & \text { Spherical stress } \\ r_{\alpha}, r_{\beta} & \text { Hankel transform parameters } \\ s & \text { Laplace transform parameter } \\ t & \text { Time parameter } \\ \chi & \text { Fractional parameter } \\ \Lambda & \text { Relaxation time parameter } \\ j, k & \text { Indexes of Fox-H function } \\ \Gamma(\boldsymbol{\square}) & \text { Gamma function } \\ \delta & \text { Convolution parameter }\end{array}$

\section{Appendix A. Finite Hankel transform}

$\int_{0}^{R}\left(\frac{\partial^{2} \overline{u_{1}}}{\partial r^{2}} r J_{1}\left(r r_{\alpha}\right)+\frac{\partial \overline{u_{1}}}{\partial r} J_{1}\left(r r_{\alpha}\right)+\overline{u_{1}} J_{1}\left(r r_{\alpha}\right)\right) d r=$

$\left(R r_{\alpha} J_{2}\left(R r_{\alpha}\right)-r_{\alpha}^{2}\right) \overline{u_{1 H}}\left(r_{\alpha}, t\right)$,

$\int_{0}^{R}\left(\frac{\partial^{2} \overline{u_{2}}}{\partial r^{2}} r J_{0}\left(r r_{\beta}\right)+\frac{\partial \overline{u_{2}}}{\partial r} J_{0}\left(r r_{\beta}\right)\right) d r=\left(R r_{\beta} J_{1}\left(R r_{\beta}\right)-\right.$

$\left.r_{\beta}^{2}\right) \overline{u_{2 H}}\left(r_{\beta}, t\right)$

$\bar{u}_{1 H}\left(r_{\alpha}, s\right)=\int_{0}^{R} \bar{u}_{1 H}\left(r_{\alpha}, s\right) r J_{1}\left(r r_{\alpha}\right) d r$,

$\bar{u}_{2 H}\left(r_{\beta}, s\right)=\int_{0}^{R} \bar{u}_{2 H}\left(r_{\beta}, s\right) r J_{0}\left(r r_{\beta}\right) d r$,

$\bar{u}_{1}(r, s)=\frac{2}{R^{2}} \sum_{\alpha=1}^{\infty} \bar{u}_{1 H}\left(r_{\alpha}, s\right) \frac{J_{1}\left(R r_{\alpha}\right)}{J_{2}^{2}\left(R r_{\alpha}\right)}, \bar{u}_{2}(r, s)=$

$\frac{2}{R^{2}} \sum_{\beta=1}^{\infty} \bar{u}_{2 H}\left(r_{\beta}, s\right) \frac{J_{0}\left(R r_{\beta}\right)}{J_{1}^{2}\left(R r_{\beta}\right)^{\prime}}$

$\frac{R^{2} J_{2}\left(R r_{\alpha}\right)}{r_{\alpha}}=\int_{0}^{R} J_{1}\left(r r_{\alpha}\right) r^{2} d r$ and $\frac{R J_{1}\left(R r_{\beta}\right)}{r_{\beta}}=$

$\int_{0}^{R} J_{0}\left(r r_{\beta}\right) r d r$,

$\frac{\partial \bar{u}_{1}(r, s)}{\partial r}-\frac{1}{r} \bar{u}_{1}(r, s)=$

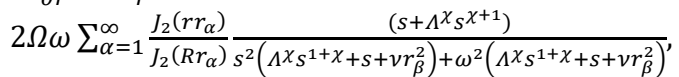

$\frac{\partial \bar{v}_{1}(r, s)}{\partial r}=\frac{2 U \omega}{R} \sum_{\beta=1}^{\infty} \frac{J_{1}\left(r r_{\beta}\right)}{J_{1}\left(R r_{\beta}\right)} \frac{\left(s+\Lambda^{\chi} s^{\chi+1}\right)}{s^{2}\left(\Lambda \chi_{S^{1+\chi}}+s+v r_{\beta}^{2}\right)+\omega^{2}\left(\Lambda \chi_{\left.S^{1+\chi}+s+v r_{\beta}^{2}\right)}\right.}$

\section{References}

Abdulhameed M, Vieru D, and Sharidan S (2016). Comparison of different pressure waveforms for heat transfer performance of oscillating flow in a circular cylinder. Engineering Science and Technology, an International Journal, 19(2): 1040-1049.

Abro KA (2016). Porous effects on second grade fluid in oscillating plate. Journal of Applied Environmental and Biological Sciences, 6(5): 17-82.

Abro KA and Solangi MA (2017). Heat transfer in magnetohydrodynamic second grade fluid with porous impacts using caputo-fabrizoi fractional derivatives. Punjab University Journal of Mathematics, 49(2): 113-125.

Abro KA, and Shaikh AA (2015). Exact analytical solutions for Maxwell fluid over an oscillating plane. Science International (Lahore), 27(2): 923-929.
Jamshoro, Pakistan for generous support and facilities of this research work.

$\begin{array}{ll}u_{2}(r, t) & \text { Oscillating velocity }\left(\mathrm{m}^{\mathrm{s}} \mathrm{s}^{-1}\right) \\ H_{j, k+1}^{1, j}(\mathrm{Z}) & \text { Fox-H function } \\ H(t) & \text { Heaviside function } \\ S & \text { Extra stress tensor } \\ \mathrm{L} & \text { Velocity gradient } \\ e_{\theta}, e_{z} & \text { Unit vectors } \\ \mu & \text { Viscosity of the fluid }(\text { Pa.s }) \\ \omega & \text { Frequency } \\ U, \Omega & \text { Non zero constants } \\ \rho & \text { Density of the fluid }\left(\mathrm{kg}^{\mathrm{m}} \mathrm{m}^{-3}\right) \\ v & \text { Kinematic viscosity }\left(\mathrm{m}^{2} \cdot \mathrm{s}^{-1}\right) \\ r & \text { Radius of cylinder }(\mathrm{m}) \\ T & \text { Transpose } \\ s_{1}, s_{2}, s_{3} & \text { Letting parameters }\end{array}$

Abro KA, Mukarrum H, and Mirza MB (2016). Impacts of magnetic field on fractionalized viscoelastic fluid. Journal of Applied Environmental and Biological Sciences, 6(9): 84-93.

Abro KA, Mukarrum H, and Mirza MB (2017a). Slippage of fractionalized Oldroyd-B fluid with magnetic field in porous medium. Progress in Fractional Differentiation and Applications: An International Journal, 3(1): 69-80.

Abro KA, Mukarrum H, and Mirza MB (2017b). Analytical solution of MHD generalized burger's fluid embedded with porosity. International Journal of Advanced and Applied Sciences, 4(7): 80-89.

Abro KA, Mukarrum H, Mirza MB, Khalil-ur-Rehman C (2017c). Flow of generalized burger's fluid in Rayleigh stokes problem. Journal of Applied Environmental and Biological Sciences, 7(5): 55-63.

Akl Y (2014). Unsteady boundary layer flow along a stretching cylinder an analytical solution. Journal of Mathematics and Statistics, 10(2): 117-124.

Altintas A and Ozkol I (2015). Magnetohydrodynamic flow of liquid-metal in circular pipes for externally heated and nonheated cases. Journal of Applied Fluid Mechanics, 8(3): $507-$ 514.

Chen C, Chen K, and Yang T (2004). Unsteady unidirectional flow of an Oldroyd-B fluid in a circular duct with different given volume flow rate conditions. International Journal of Heat and Mass Transfer, 40(3-4): 203-209.

Erdogan M and Imrak C (2005). On unsteady unidirectional flows of a second grade fluid. International Journal of Nonlinear Mechanics, 40(10): 1238-1257.

Fetecau C and Corina F (2005). Starting solutions for some unsteady unidirectional flows of a second grade fluid. International Journal of Engineering Science, 43(10): 781-789.

Fetecau C, Fetecau C, and Vieru D (2007). On some helical flows of Oldroyd-B fluids. Acta Mechanica, 189(1-4): 53-63.

Fetecau C, Mahmood A, and Jamil M (2010). Exact solutions for the flow of a viscoelastic fluid induced by a circular cylinder subject to a time dependent shear stress. Communication in Nonlinear Science and Numerical Simulation, 15(12): 39313938.

Fetecau C, Mahmood A, Fetecau C, and Vieru D (2008). Some exact solutions for the helical flow of a generalized Oldroyd-B fluid 
in a circular cylinder. Computers with Mathematics and Applications, 56(12): 3096-3108.

Hayat T, Khan M, and Ayub M (2004a). On the explicit analytic solutions of an Oldroyd 6-constant fluid. International Journal of Engineering Science, 42(2): 123-135.

Hayat T, Khan M, and Ayub M (2006). Some analytical solutions for second grade fluid flows for cylindrical geometries. Mathematical and Computer Modelling, 43(1): 16-29.

Hayat T, Khan M, Siddiqui AM, and Asghar S (2004b). Transient flows of a second grade fluid. International Journal of Nonlinear Mechanics, 39(10): 1621-1633.

Jamil M, Rauf A, Fetecau C, and Khan NA (2011). Helical flows of second grade fluid due to constantly accelerated shear stresses. Commun Nonlinear Sci Numer Simulat, 16(4): 19591969.

Mahmood A, Fetecau C, Khan N, and Jamil M (2010). Some exact solutions of the oscillatory motion of a generalized second grade fluid in an annular region of two cylinders. Acta Mechanica Sinica, 26(4): 541-550.

Malekzadeh A, Heydarinasab A, and Bahram D (2011). Magnetic field effect on fluid flow characteristics in a pipe for laminar flow. Journal of Mechanical Science and Technology, 25(2): 333-339.

Masood K, Rabia M, and Hussain M (2016). Nonlinear radiative heat transfer to stagnation-point flow of Sisko fluid past a stretching cylinder. American Institute of Physics Advances, 6(5): 1-10.

Muhammad J, Abro KA, and Khan NA (2015). Helices of fractionalized Maxwell fluid. Nonlinear Engineering, 4(4): 191-201.

Nazar M, Fetecau C, and Awan A (2010). A note on the unsteady flow of a generalized second grade fluid through a circular cylinder subject to a time dependent shear stress. Nonlinear Analysis: Real World Applications, 11(4): 2207-2214.
Rashidi MM, Ali M, Freidoonimehr N, Rostami B, and Anwar MH (2014). Mixed convective heat transfer for MHD viscoelastic fluid flow over a porous wedge with thermal radiation. Advances in Mechanical Engineering. Available online at: http://journals.sagepub.com/doi/abs/10.1155/2014/73593

Rashidi MM, Mohamed A, Rostami B, Rostami P, and Xie GN (2015). Heat and mass transfer for MHD viscoelastic fluid, flow over a vertical stretching sheet with considering soret and dufour effects. Mathematical Problems in Engineering, 2015: Article ID 861065, 12 pages. https://doi.org/10.1155/2015/861065

Rashidi MM, Momoniat E, and Rostami B (2012). Analytic approximate solutions for MHD boundary-layer viscoelastic fluid flow over continuously moving stretching surface by homotopy analysis method with two auxiliary parameters. Journal of Applied Mathematics, 2012: Article ID 780415, 19 pages. https://doi.org/10.1155/2012/780415

Rostami B, Rashidi MM, Rostami P, Momoniat E, and Freidoonimehr N (2014). Analytical investigation of laminar viscoelastic fluid flow over a wedge in the presence of buoyancy force effects, 2014: Article ID 496254, 11 pages. https://doi.org/10.1155/2014/496254

Shah M and Qi H (2010). Starting solutions for a viscoelastic fluid with fractional burgers model in an annular pipe. Nonlinear Analysis: Real World Applications, 11(1): 547-554.

Siddique I and Vieru D (2009). Exact solution for the rotational flow of a generalized second grade fluid in a circular cylinder. Acta Mechanica Sinica, 25(6): 777-785.

Sulochana C and Sandeep N (2016). Flow and heat transfer behavior of MHD dusty nanofluid past a porous stretching/shrinking cylinder at different temperatures. Journal of Applied Fluid Mechanics, 9(2): 543-553.

Wang S and Xu M (2009). Axial couette flow of two kinds of fractional viscoelastic fluids in an annulus. Nonlinear Analysis: Real World Applications, 10(2): 1087-1096. 